\title{
COUPLED TORSIONAL CANTILEVERS FOR LABEL-FREE SINGLE MOLECULAR LEVEL BIO-DETECTION AND NANOMATERIALS CHARACTERIZATION
}

\author{
Ozgur Sahin \\ Rowland Institute at Harvard, Cambridge, \\ MA USA \\ Henrik H. J. Persson \\ Stanford Genome Technology Center, Palo \\ Alto, CA USA \\ Calvin F. Quate, and Olav Solgaard \\ E. L. Ginzton Laboratory, Stanford, CA
}

\begin{abstract}
We have developed a micromachined atomic force microscope cantilever with an integrated force sensor that enables time-resolved measurement of forces between the vibrating tip and the sample in tapping-mode atomic force microscopy. These tip-sample interaction forces depend on the elastic, viscoelastic, and adhesive properties of the sample and are determined by the chemical and structural composition of the material. With this technique we are able to detect biomolecular associations on the molecular level and map chemical compositional variations and physical changes within materials at the nanoscale.
\end{abstract}

\section{INTRODUCTION}

Mechanical properties of nanoscale objects highly depend on their chemical composition. Measurement of nanomechanical properties of materials and macromolecules will allow us to map chemical compositional variations and detect biomolecular reactions. Tapping-mode atomic force microscopy provides a framework to interact with nanoscale objects mechanically and non-destructively. In this mode of atomic force microscopy the cantilever with the sharp tip is vibrating on resonance in the vicinity of the sample surface so that the tip intermittently interacts with the surface. Dynamic forces between the tip and the sample carry information on the local mechanical properties with nanoscale spatial resolution. Unfortunately, in conventional tapping mode, measurement of these dynamic forces with temporal resolution is not possible as the cantilever oscillation depends only on the time average value of tip sample forces $[1,2]$. Recently, measurement of higher harmonics of tip-sample forces has been demonstrated [3-6]. These harmonic forces carry information on the time variation of tip-sample forces, however, they have limited signal to noise ratios. Enhancement of a particular higher harmonic signal has been demonstrated with the use of special micromachined cantilevers [5], however that technique recovers only one higher harmonic.

Here we present a micromachined atomic force microscope cantilever, the coupled torsional cantilever, which allows us to simultaneously measure higher harmonics of tipsample forces up to the $20^{\text {th }}$ harmonic.

\section{THE COUPLED TORSIONAL CANTILEVER}

The coupled torsional cantilever has a tip that is offset from the longitudinal axis (Fig. 1 (a)). When the cantilever is tapping on a sample while vibrating in its first flexural mode, offset tip couples the tip-sample forces to the first torsional mode (Fig. 1(b)). The first torsional vibration mode has a much larger bandwidth and mechanical response at high frequencies than the first flexural vibration mode (Fig. 2(a)). The coupling to the torsional mode therefore provide the high-bandwidth force sensor that is required to detect the tip-sample interaction forces that are of short duration compared to the fundamental vibration frequency. The quadrant position sensitive detectors used in commercial atomic force microscopes allow us to measure torsional and flexural deflections of the cantilever simultaneously. The simultaneously obtained flexural and torsional vibration spectra of a cantilever tapping on a polystyrene sample (Fig. 2(b)) show that the torsional vibrations enhance the signal levels at higher harmonics by 20 to $40 \mathrm{~dB}$. It is these higher harmonics that contain information about the high speed variation of tip-sample forces within a period of cantilever oscillation. Therefore, coupled torsional cantilevers recover the information on time-resolved tip-sample forces.
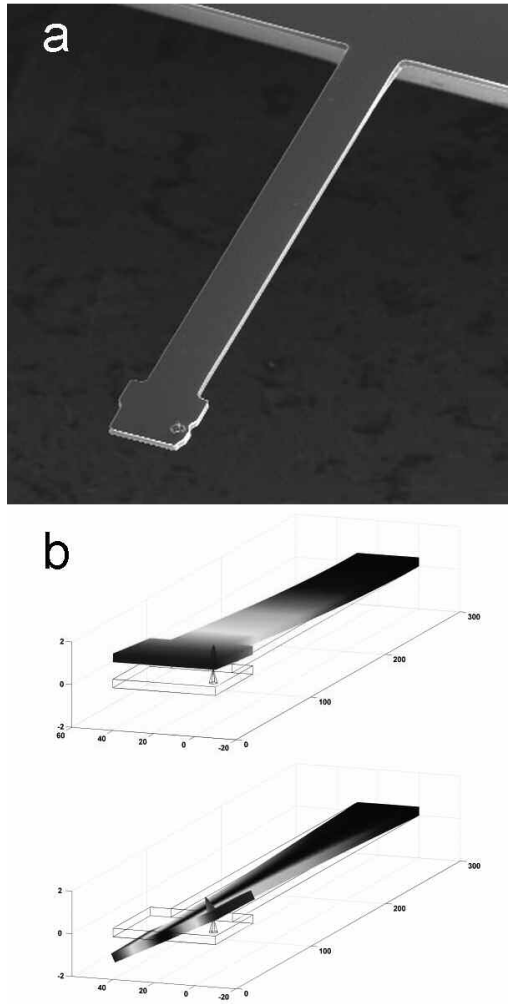

Figure 1. (a) SEM micrograph of a coupled torsional cantilever. The cantilever is $300 \mathrm{um}$ long, $30 \mathrm{um}$ wide, and 3 um thick. Note the offset location of the sharp tip. (b) Simulated mode shapes of first flexural and torsional resonances. 

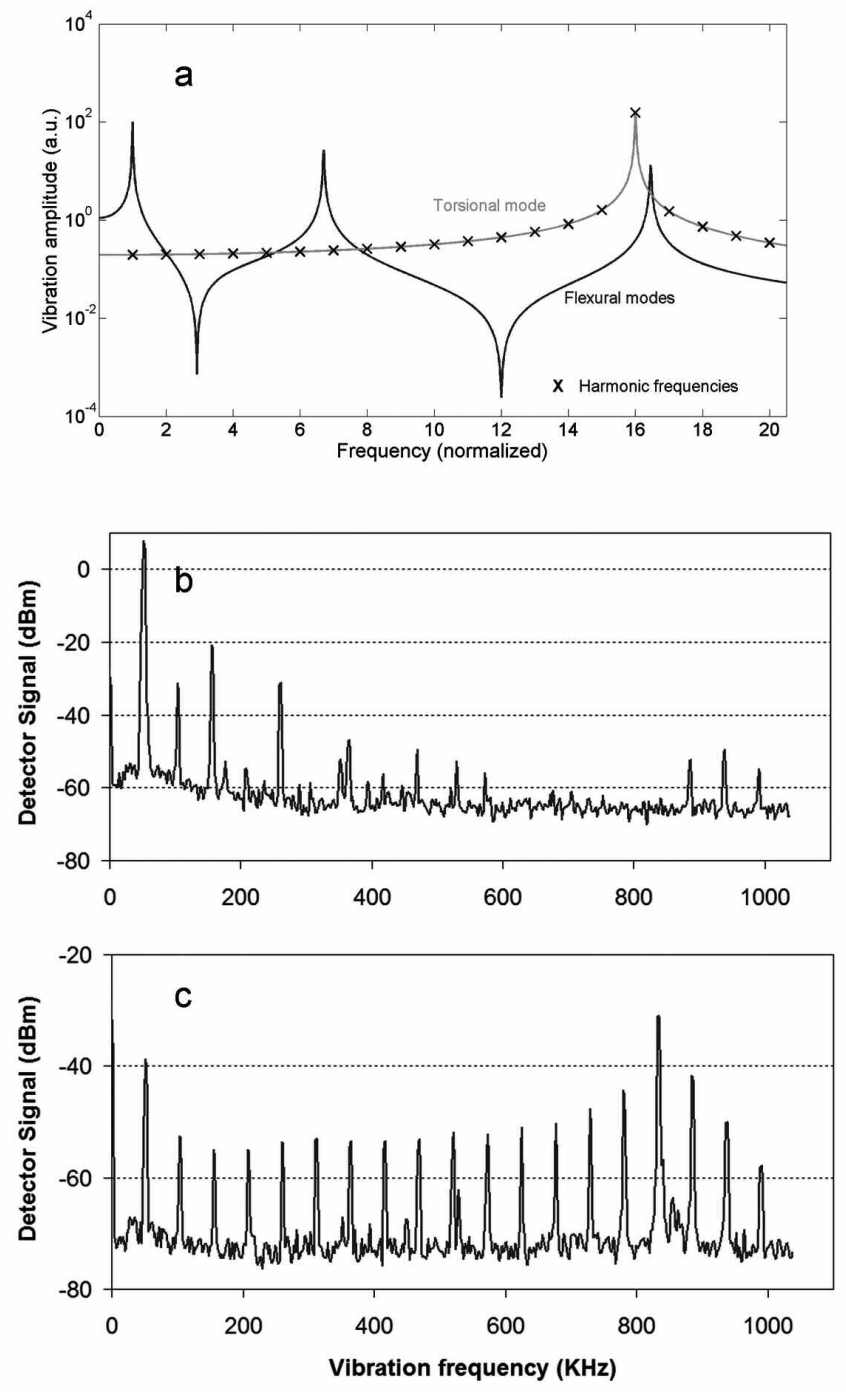

Figure 2. (a) Frequency response of the flexural and torsional vibrations of a typical rectangular cantilever. Measured flexural (b) and torsional (c) vibration spectra of a coupled torsional cantilever tapping on a polystyrene sample.

\section{TIME RESOLVED INTERACTION FORCE MEASUREMENTS}

The ability to measure higher harmonics of tip-sample forces allows us to reconstruct the periodic tip-sample force waveform. Those frequency components of tip-sample forces that are close to the torsional resonance frequency get enhanced by the resonance. This is seen in the torsional vibrations given in Fig. 2(b). The harmonics that are close to $850 \mathrm{KHz}$ have larger magnitudes. We are interested in the actual magnitude of the forces. Therefore, we correct for the resonance enhancement of the first torsional mode. This is done by sampling the torsional deflection signal at a high sampling rate (with a digital oscilloscope) and then passing it through a linear filter that has a transfer function equal to the inverse of the first torsional mode. We recorded torsional oscillations on three different materials (high density polyethylene, low density polyethylene, and highly oriented pyrolytic graphite) and calculated time resolved tipsample forces. The resulting waveforms are given in figure 3(a). These measurements are recorded under the same drive force and tapping amplitude, and with the same tip. These waveforms show that tip-sample interaction forces are different for different materials. Interpretation of the differences is difficult, however. The interpretation is simplified when the the forces are plotted against tip-sample separation (Fig. 3 (b)). Note that the tip is approaching and retracting from the surface in a sinusoidal orbit and the peak forces are obtained when the tip is at the bottom of its trajectory. The slopes of the curves in Figure 3(b) at negative separations (sample indentation) are proportional to the stiffness of the sample. As expected, the slope on graphite is higher than the polymers and the slope on the high density polyethylene is higher than the low density polyethylene. An interesting feature of these plots is the hysteresis they exhibit. Hysteresis in the force vs. distance plots is a measure of adhesiveness. As a result of attractive forces, compliant materials can be pulled above its equilibrium surface level.
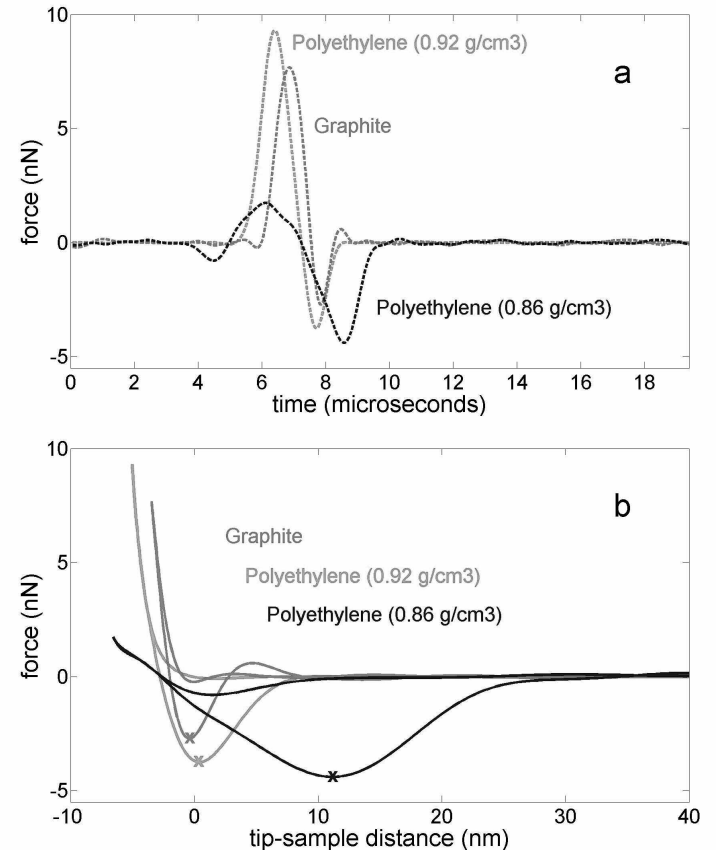

Figure 3. (a) Time-resolved tip-sample forces measured with coupled torsional cantilever on three materials. (b) The same measurements in (a) are plotted against tip-sample separation. Negative separation corresponds to sample indentation. The slopes depend on material stiffness. Hysteresis and maximum negative forces depend on the adhesiveness of the sample.

\section{OBSERVING PHASE TRANSITIONS OF COMPOSITE POLYMERS}

We studied thermal characteristics of an ultra-thin binary polymer blend film with sub-micron features. The polymer is composed of polystyrene and PMMA. The latter has a higher 
glass transition temperature. We imaged mechanical properties at the surface at elevated temperatures with our torsional harmonic technique (Fig. 4 (a)). In this technique, we scan the surface in tapping mode and simultaneously record a particular higher harmonic signal in the torsional spectrum. Higher harmonic imaging has been previously demonstrated with the use of harmonic signals in the flexural response with lower signal to noise levels [3-5]. The advantage of using torsional harmonics is that one can lock onto any higher harmonic, because all the harmonics provide good signal levels. Note that the information in each harmonic is not the same [7].

a

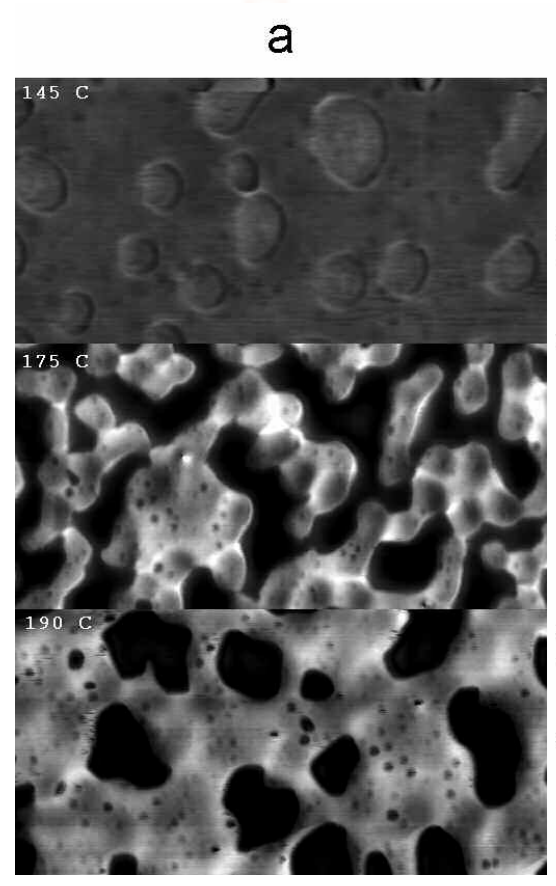

At lower temperatures, the two polymers have similar characteristics. However, as the glass transition temperature of polystyrene is exceeded, we observed a drastic change in the contrast indicating softening in the polystyrene regions. Detailed understanding of the changes is obtained with the measurement of time resolved forces on both materials. Figure 4 (b) shows the time-resolved force measurements plotted against tip-sample separation on polystyrene and PMMA. These plots show that polystyrene is softening significantly as the temperature is increased, while PMMA does not exhibit detectable changes. These measurements show that polystyrene regions are going through glass transition. To our knowledge, this is the first observation of glass transition in polymers at the nanoscale.
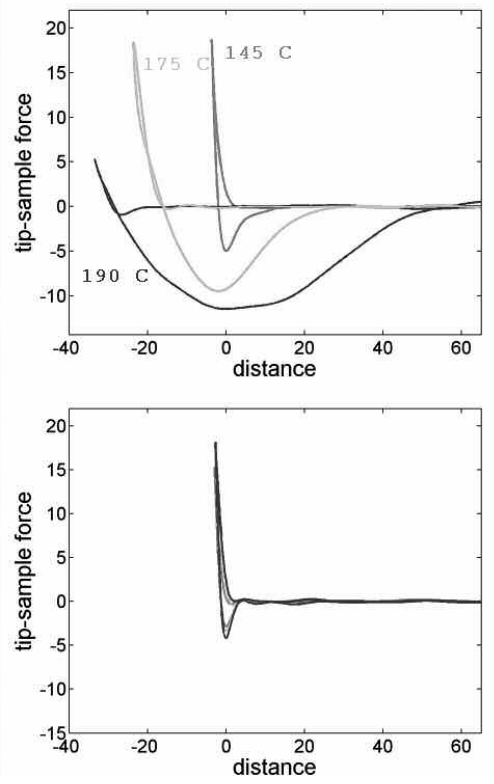

Figure 4. (a) Harmonic force images of a thin blended binary polymer film at three temperatures obtained at the $16^{\text {th }}$ harmonic. Increasing contrast suggests that one polymer component has gone through glass transition. Scan area is $2.5 \times 5$ microns at $145^{\circ} \mathrm{C}$ and 5 by 10 microns at $175^{\circ} \mathrm{C}$ and $190^{\circ} \mathrm{C}$. (b) Time-resolved tip-sample force measurements on the two material components reveal the nature of the changes. One material is softening drastically and becoming adhesive. The other material is nearly intact.

\section{DISCRIMINATION OF SINGLE HYBRIDIZED DNA MOLECULES}

An application of timeresolved AFM technology is to detect biomolecular associations through mechanical changes in molecules. We studied mechanical changes in the hybridized DNA-oligonucleotides versus single stranded DNA. A mixture of hybridized and single stranded molecules are immobilized on a gold surface with thiol linkers [8] and imaged in air with our technique. We employed different mixture rates to obtain control groups in the experiments. In the first sample only single stranded DNA molecules are immobilized. In the second sample $0.1 \%$ of the molecules in the mixture were double stranded. In the third sample all the molecules were double stranded. We recorded harmonic images with the use of a resonantly enhanced $16^{\text {th }}$ flexural harmonic. These images are given in figure 5. The images show good contrast between the fully hybridized and fully single stranded samples. Furthermore, on the sample with $0.1 \%$ hybridization we observe features with approximately 40 $\mathrm{nm}$ in diameter that we believe correspond to individual hybridized DNA molecules. The increased radii of these features are due to tip convolution. This hypothesis is supported by the comparison of signal levels measured on these features. On the right side of each harmonic image, we give actual harmonic signal levels measured at a section of the image. The signal level observed on the fully single stranded sample $(\sim 0.9 \mathrm{mV})$ is also observed on the $0.1 \%$ percent hybridized sample as the background signal levels. On the bright features in this image, the harmonic signal drops to $0.45 \mathrm{mV}$. This signal level is also recorded on the bright features of the fully hybridized sample. These results indicate that bright features with signal levels around $0.45 \mathrm{mV}$ correspond to hybridized molecules. 
Furthermore, the hybridization of a single DNA molecule results in a contrast that provides approximately $30 \mathrm{~dB}$ signal to noise ratio. The high resolution of the AFM tip allows us to see individual hybridized molecules, and makes it possible to count the number of molecules. Our results show that with this technique, it is possible to quantitatively measure hybridization reactions with large dynamic range and detect very low concentrations of molecules. Alternative technologies like nanowire based electrical sensors, or fluorescence and plasmon based optical sensors do not provide this level of sensitivity, and neither do they provide the spatial resolution to identify individual molecules. Another advantage of this technique is that the measurements are performed on label-free DNA molecules and the recorded signal levels are specific to the chemical species. Other label-free techniques, such as resonant mass sensors, do not provide chemical specificity, since any attachment of a foreign body on the resonating device will result in a shift in resonance frequency. a
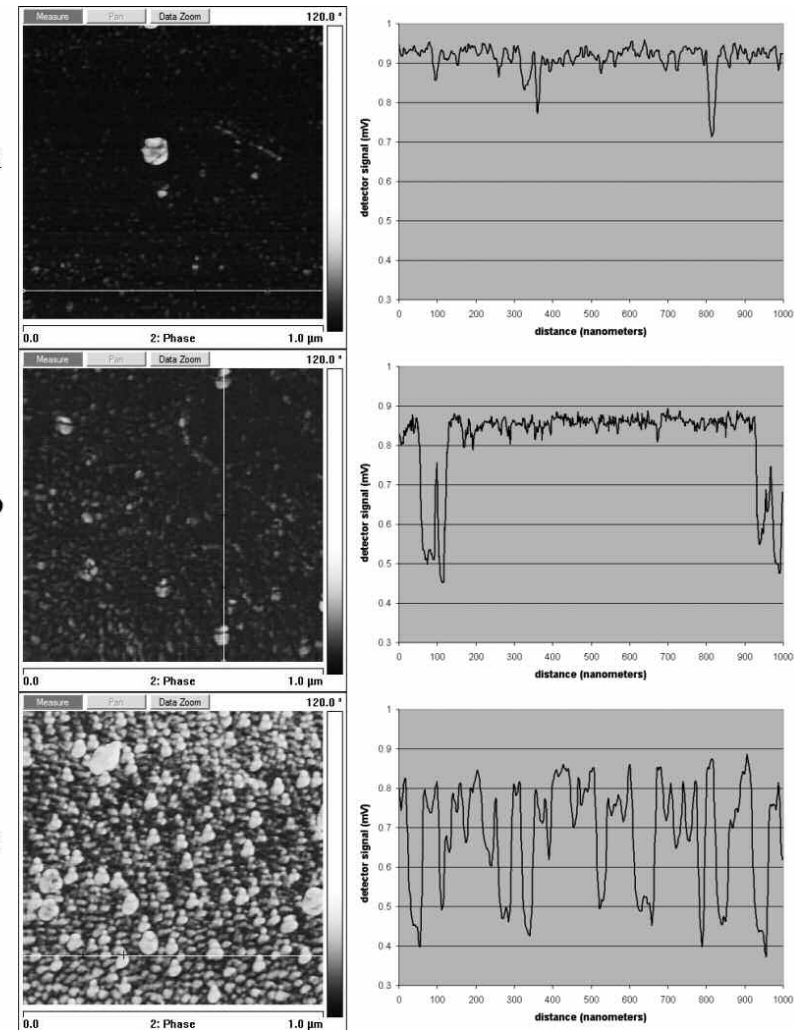

Figure 5. Harmonic force images obtained on self assembled DNA oligo-nucleotides on atomically flat gold. Scan areas are 1 um by lum. Signal levels recorded on a section of the images are given to the right of the corresponding image. The three samples are prepared by immobilizing a mixture of 20 nucleotide long single and double stranded DNA molecules. Percentage of the double stranded molecules in the mixture are $0 \%$ in (a), $0.1 \%$ in (b), and $100 \%$ in (c). The signal levels given to the right of each image show that single stranded molecules result in a signal level of $0.9 \mathrm{mV}$ and double stranded molecules result in $0.45 \mathrm{mV}$. The widths of the features in (b) suggest that these belong to individual hybridized DNA molecules, since our tips are typically 20-50 $\mathrm{nm}$ wide.

\section{CONCLUSIONS}

We presented the coupled torsional cantilever to measure the mechanical interaction between the sharp tip of the atomic force microscope and the sample. Mechanical properties of materials and individual molecules highly depend on the physical and chemical properties. We used this fact to study phase transitions of composite polymers with nanometer level spatial resolution and observed the glass transition of submicron polystyrene domains within the composite. Then we studied the effects of hybridization of DNA molecules on the mechanical properties of the surface and used this phenomenon to detect and quantify hybridized DNA molecules. This technology shows promise for nanomaterials characterization and highly sensitive and quantitative biomolecular analysis.

\section{REFERENCES}

[1] A. S. Paulo and R. Garcia, "Unifying theory of tapping-mode atomic force microscope" Phys. Rev B. 66, 041406(R) (2002).

[2] J. P. Cleveland, B. Anczykowski, A. E. Schmid, and V. B. Elings, "Energy dissipation in tapping-mode atomic force microscopy.” Appl. Phys. Lett. 72, 2613-2615 (1998).

[3] R. Hillenbrand, M. Stark, and R. Guckenberger, "Higherharmonics generation in tapping-mode atomic force microscopy: Insights into tip-sample interaction." Appl. Phys. Lett. 76, 34783480 (2000).

[4] R. W. Stark and W. M. Heckl "Higher harmonics imaging in tapping-mode atomic-force microscopy." Rev. Sci. Instrum. 74, 5111-5114 (2003).

[5] O. Sahin, G. Yaralioglu, R. Grow, S. F. Zappe, A. Atalar, C. F. Quate and O. Solgaard, "High resolution imaging of elastic properties using harmonic cantilevers." Sensors and Actuators A, 114, 183-190 (2004).

[6] M. Stark, R. W. Stark, W. M. Heckl and R. Guckenberger, "Inverting dynamic force microscopy: from signals to time resolved forces.” PNAS, 99, 8473-8478 (2002).

[7] O. Sahin, A. Atalar, C. F. Quate, and O. Solgaard, "Resonant harmonic response in tapping-mode atomic force microscopy." Phys. Rev. B. 69165416 (2004)

[8] T. M. Herne and M. J. Tarlov: Characterization of DNA probes immobilized on gold surfaces. J. Am. Chem. Soc. 119, 8916-8920 (1997) 